\title{
THE PREDICTIVE ABILITY OF TYPE D PERSONALITY PATTERN, ANXIETY, AND DEPRESSION IN CARDIAC DISEASE**
}

(Received: 2 December 2020; accepted: 31 July 2021)

\begin{abstract}
A growing body of empirical evidence suggests psychological and personality risk factors for cardiovascular disease in Western developed countries. This study expands that line of health research to a community sample of 309 Jordanians (half of whom were diagnosed with heart problems). Using the Distress Scale, Beck Depression Inventory-II, and State-Trait Anxiety Inventory, this study determined that Type D personality, depression, and anxiety have value in predicting cardiovascular disease. The results showed that older individuals with high levels of social inhibition are more likely to have heart disease compared to younger participants. Anxiety and depression were also potent risk factors. The emerging pattern confirms the cross-cultural validity of Type $\mathrm{D}$ personality as well as depression and anxiety indices in predisposing individuals to cardiovascular disease. This study calls for using a multiple-level-analysis approach combining personality and social influences. Patients and health providers can engineer health through psychological wellness and health-promotive behavior. Programs based on self-empowerment theory that target the roots of anxiety and depression, as well as the social inhibition and negative affectivity dimensions of Type D personality (e.g., rage, hostility), should be an integral component of any therapy or intervention.
\end{abstract}

Keywords: Type D personality; cardiovascular disease; depression; anxiety

\section{Introduction}

Type D (distressed) personality has been examined as a risk factor for both increased morbidity and mortality in various chronic illnesses. First introduced by DeNOLLET

* Corresponding Author: Wisam Breik, Al-Ahliyya Amman University, Department of Psychology, Amman 19328, Jordan; wbreik@ammanu.edu.jo.

** Ethical guidelines followed the World Medical Association (2013) and International Committee of Medical Journal Editors (2018). 
and colleagues (1995), Type D has since been identified by its implications in studies of patients with coronary heart disease (CHD), or coronary artery disease. The significance of Type D personality in patients with CHD is the basis of this present study. We sought to determine whether Type D personality, depression, and anxiety have a value in predicting cardiovascular disease.

The Type $\mathrm{D}$ personality construct is defined by elevated levels of negative affectivity and social inhibition. Negative affectivity is the tendency to experience negative emotions and is associated with a susceptibility to depression and anxiety. Social inhibition is the tendency to inhibit emotional expression. It is typified by increased feelings of interpersonal insecurity and stress, as well as rigid control of self-expression to avoid perceptions of disapproval by others. Negative affectivity correlates positively with neuroticism on the five-factor model of personality, social inhibition correlates negatively with extraversion, and both negative affectivity and social inhibition correlate negatively with conscientiousness. Assessment of this particular personality construct is often made through the personality Distress Scale (DS14; Denollet 2005). To be classified as Type D, an individual must exceed the standardized cut-off score $\geq 10$ in both negative affectivity and social inhibition subscales.

Individuals with Type D personality are prone to elevated levels of psychological distress, characterized by a propensity towards worry, unhappiness, insecurity, pessimism, feelings of tension around others, unwillingness to interact in social settings, and self-blame. Type D patterns also may correlate to low self-esteem, depression, difficulties perceiving and utilizing social support, exhaustion, and minimal satisfaction with quality of life. Major depression and anxiety disorders have been determined to be effective risk factors for CHD (DE HeRT et al. 2018). In comparison to nondepressed individuals, individuals diagnosed with depression are at higher risk of developing clinical coronary disorders, and major studies linked depression with all-cause mortality (ANGERMAnN et al. 2011; De Hert et al. 2018; MORASKA et al. 2013).

The prevalence of Type D personality is $21 \%$ in the general population, yet 28-53\% among cardiac patients (DENOLLET 2005). Generally, biological and behavioral risk mechanisms are linked to Type D personality which predispose individuals to chronic illness, increased levels of maladaptive health behaviors, lower levels of social functioning, and adaptation of poor coping strategies activation (DENOLLET et al. 2008). The disuse of social support as a means of reducing psychological distress should be taken into particular regard. Type D individuals fail to perceive the social support available to them (GINTING et al. 2016). Inadherence to medication regimens and other treatment was also notably higher in Type D patients (WiLLIAMs et al. 2011; Wu et al. 2015). Type D has been consistently linked to unhealthy eating behaviors (BоотH \& Williams 2015; NeFs et al. 2015) and lack of motivation to exercise (Bunevicius et al. 2014; Wiencierz \& Williams 2017). Thus, Type D personality traits may lead to adverse health outcomes in various chronic illnesses. 
Findings from past studies have established the relationship between psychological factors and adverse prognosis in patients suffering from heart disease (DENOLLET 2005). Thus, recognizing the function of Type D personality risk is important not only for implementing strong proactive and reactive intervention programs but also in understanding the etiology of cardiovascular disease. Risk studies in general are grounded in epidemiology and serve as the foundation of health-promotive behavior, with emphasis on the perception of disease patterns across populations and variables that steer the course and development of these patterns (LILIENFELD \& LILIENFELD 1980). The individual may alter the course of disease. Risk may be actively reduced or inverted into assets through proactive preventive healthcare offered to susceptible populations (primary and secondary care), as well as through responsive programs for those already diagnosed with cardiovascular conditions (tertiary care).

\section{Methodology}

We present a comprehensive take on the evaluation of potential psychological and personality mechanisms linking Type $\mathrm{D}$, depression, and anxiety to cardiac disease, emphasizing the heterogeneity of plausible pathophysiological pathways. Data from 309 subjects in Jordan were collected to answer the following research questions:

1. What is the predictive ability of a model to identify patients with heart disease compared to a comparative group without heart disease, based on three factors: Type D personality, depression, and anxiety?

2. Does Type D personality mediate the relationship between cardiac disease and the outcome variables as measured by depression and anxiety?

\subsection{Sample}

This study included 309 subjects, $54 \%(n=166)$ males. The age range was $19-82$ with a mean of 45 years $(S D=12.07)$. Of the subjects, 156 were diagnosed with a cardiovascular disease marker (blood vessel diseases, coronary artery disease, arrhythmias, angina, or stroke) a year or more prior to the study. Other heart conditions, including congenital heart defects, were excluded. Patients were included in the study if they have been diagnosed for at least one year, in order to be considered stable, with any of the CHD markers (blood vessel diseases, coronary artery disease, arrhythmias or angina). Other heart conditions affecting the heart muscle, valves, or rhythm, including congenital heart defects, were excluded.

The comparative group $(n=153)$ had no history of cardiovascular disease, hypertension, high cholesterol, or other life-threatening diseases (e.g., cancer, autoimmune diseases) and excluded people who could not read and write. The non-patient group was recruited from the same hospitals, were hospital employees, or had different 
vocations in Amman. The groups had similar demographic characteristics (patient and nonpatient group with $54 \%$ and $53 \%$ males, respectively and mean age, $M=$ 42 and 48 years old, respectively). Both groups were recruited over the same three months. Participation was anonymous and voluntary, with no financial compensation offered. Participants signed a written informed consent form.

\subsection{Instruments}

\subsubsection{Demographic questionnaire}

All participants completed the demographic questionnaire (age and gender) and provided lifestyle information such as smoking, and their type of heart condition: blood vessel diseases, coronary artery disease, arrhythmias, angina, or stroke.

\subsubsection{The DS14}

The DS14 is a 14-item questionnaire with two seven-item subscales (DeNOLLET 2005), which measures the tendency of negative emotions (negative affectivity) and inhibited self-expression in social interactions (social inhibition). Participants respond on a 5-point Likert scale from 0-4 to a range of statements such as 'I am often in a bad mood' (negative affectivity) and 'I find it hard to start a conversation' (social inhibition). The total scores range from $0-28$ for each subscale. According to DenOLlet (2005), a score of 10 or more on both scales is used to classify respondents as having a Type D personality. DenOLlet (2005) derived the cut-off value from the median split in representative samples on negative affectivity and social inhibition scores of participants in his study; moreover, clinical evidence for this cut-off value-based classification was obtained in longitudinal clinical studies, and empirical evidence was obtained from a latent class cluster analysis (EMONs et al. 2007). Test-retest reliability of $r=0.72$ for the negative affectivity and 0.82 for the social inhibition subscales was reported as well as a Cronbach's alpha of 0.88 for the negative affectivity and 0.86 for the social inhibition subscales (DENOLLET 2005).

The DS14 has been validated in countries around the world (Lim et al. 2011; RAZZINI et al. 2008; SvanSDOTTIR et al. 2012). The negative affectivity subscale had a high positive correlation with neuroticism $(r=0.80)$ and rehearsal $(r=0.58)$, whereas social inhibition was negatively correlated with extraversion $(r=-0.65)$ and positively correlated with emotional inhibition $(r=0.50)$, which further supports the divergent validity of the Type D factors and their individual attributes (SvANSDOTTIR et al. 2012). Negative affectivity scores had a high correlation with anxiety, depression, and stress scores, indicating that these clearly measure an increased negative effect. 
Principal axis factor analysis revealed internally consistent negative affectivity and social inhibition factors, and a confirmatory factor analysis confirmed the two-factor structure of the original scale (DENOLLET 2005) in a large sample of Icelandic cardiac patients (SvanSDOTTIR et al. 2012).

The internal consistency was also measured in the Jordanian study sample. Cronbach's alpha was 0.81 for negative affectivity and 0.85 for the social inhibition subscale. Pearson's correlation was 0.82 for negative affectivity and 0.84 for social inhibition. An English form of the DS14 was translated into Arabic by two bilingual psychologists who independently translated the Arabic form of DS14 items from Arabic into English to ensure the accuracy of the translation. The two independent translations were examined, and one version was constructed. Two psychologists were asked to review the translated version to ensure the accuracy of the wording. Internal consistency estimates in this sample were lower than DeNOLLET's (2005). Cronbach's alpha was 0.75 for Negative Affectivity and 0.51 for Social Inhibition.

\subsubsection{State-Trait Anxiety Inventory (STAI)}

The STAI was developed by Spielberger, Gorsuch, and Lushene (1970). Forty self-report items measure two types of anxiety: state anxiety, or anxiety about an event, and trait anxiety, or anxiety level as a personal characteristic. Each scale has 20 items rated on a 4-point Likert scale of $0-4$. The score on each subscale ranges from 20-80. Higher scores indicate higher levels of anxiety.

The reliability and validity of the STAI were assessed in previous studies by SPIELBERGER and colleagues (1970), and test-retest correlations were calculated to be 0.54 for the State section and 0.86 for the Trait section. Trait anxiety can be defined as feelings of stress, worry, and discomfort that one experiences daily. This is usually perceived as how people feel across typical situations that everyone experiences daily (Spielberger \& Sydeman 1994). The Trait Anxiety subscale evaluates relatively stable aspects of anxiety proneness, including general states of calmness, confidence, and security.

A validated Arabic version (Al-Buhairi 2005) of the State Trait Anxiety Inventory-form Y (STAI-Y) (SPILBERGER et al. 1970) was adopted. Correlations between STAI-Y scores and several criteria indicated strong concurrent validity, namely, with the Catl Scale for Anxiety ( $r=0.62$ for males and 0.70 for females) and the Eisenck Scale for Neuroticism ( $\mathrm{r}=0.50$ for males and 0.60 for females). The reliability of the STAI-Y was also confirmed; Pearson's correlation coefficient on a test-retest of the same subjects in varying periods of time amounting to one day after the first application, then a week then a month, ranged between 0.77-0.87). The internal consistency was also measured in the study sample. Cronbach's alpha was 0.72 for the Trait Anxiety subscale, and the Pearson correlation was 0.83 . 


\subsubsection{Beck Depression Inventory-II (BDI-II)}

The BDI-II is a 21-item self-report instrument intended to assess the existence and severity of symptoms of depression in psychiatrically diagnosed adults and adolescents (BECK et al. 1996).

It is the most widely used instrument for detecting depression, taking only five minutes to complete. Each item is followed by a 4-point scale of 0-3. Two items have seven options indicating either an increase or decrease of appetite and sleep. A total score of $0-13$ is considered minimal range, $14-19$ is mild, 20-28 is moderate, and 29-63 is severe (BECK et al. 1996). For the current study, score reliability was $0.86,95 \% \mathrm{CI}=[0.82,0.89]$. The BDI has been reported to be highly reliable regardless of the population. It has a high coefficient alpha $(0.80)$, its construct validity has been established, and it is able to differentiate depressed from nondepressed patients. For the BDI-II, the coefficient alphas were 0.92 for outpatients and 0.93 for college students; stability coefficient reached 0.93 , which was significant at $p<$ 0.001 . The convergent validity of the BDI-II was assessed by administration of the BDI-1A and the BDI-II to two subsamples of outpatients $(N=191)$. The order of presentation was counterbalanced, and at least one other measure was administered between these two versions of the BDI, yielding a correlation of $0.93(p<0.001)$ and means of $18.92(S D=11.32)$ and $21.89(S D=12.69)$, the mean BDI-II score being 2.96 points higher than the BDI-1A. A calibration study of the two scales was also conducted. Factorial validity has been established by the intercorrelations of the 21 items calculated from the sample responses. The BDI-II has been validated in several Arabic countries (ALANSARI 2005) and is regarded as being valid and reliable, with a reported Cronbach's Alpha of 0.83 and a test-retest reliability of $r=0.74$ (Al-MusAWI 2001). A validated Jordanian version was used in the study (AL-DA'ASIN 2004). The concurrent validity of the BDI-II was confirmed in Jordanian samples; the correlation coefficients between the BDI-II and several criteria ranged between 0.71 and 0.89 . Moreover, significant differences were found in performance on the BDI between the sample of mentally ill patients and community samples. The internal consistency was also measured in the Jordanian study sample; Cronbach's alpha $(n=156)$ was 0.84 , and the Pearson correlation $(n=30)$ was 0.88 .

\subsection{Procedure}

After obtaining permission from the Jordanian Ministry of Health, three main hospitals were selected to collect information from the cardiac clinics in these hospitals. The CHD sample was randomly selected with the help of the nurses from among cardiac patients who have odd queue numbers in the reception room. All procedures performed were in accordance with the ethical standards of the institu- 
tional or national research committee in Jordan (Al-OMARI \& Al-HusSAIni 2017) and with the 1964 Helsinki declaration and its later amendments (World Medical Association 2013) or comparable ethical standards. Informed consent was obtained from all individual participants included in the study. The sample of nonpatients was randomly selected from individuals in the hospitals at the same time as the information was collected from the CHD patients. The rest were randomly selected from the employees in the hospital and different vocational sectors in Amman.

All surveys were completed in the presence of the researchers, who stayed with the participants to answer any questions. After the application was completed, all the instruments were reviewed; none were excluded because all were complete and appropriate for the purposes of the study. To ensure anonymity, participants were instructed not to provide any background information that might disclose their identities or their families'. Finally, participants received no compensation for participation in the study.

\section{Data analysis}

The results of the logistic regression analysis (see Table 1) showed four factors as significant predictors of heart problems. These factors were age, Wald $(d f=1)=$ 14.67, $p<0.001$; social inhibition, $\operatorname{Wald}(d f=1)=15.21, p<0.001$; anxiety, Wald $(d f$ $=1)=5.38, p<0.05$; and depression, $\operatorname{Wald}(d f=1)=8.89, p<0.05$. These results showed that older participants with higher levels of social inhibition, anxiety, and depression were more likely to experience heart problems.

Table 1

The results of logistic regression

\begin{tabular}{lccccccc}
\hline Factor & $B$ & Wald & $d f$ & $P$ & $-22 L$ & $R^{2}$ & Odds ratio \\
\hline Age & 0.04 & 14.66 & 1 & 0.000 & 410.51 & $0.056-0.075$ & 1.04 \\
Social Inhibition & -0.12 & 15.21 & 1 & 0.000 & 393.84 & $0.106-0.141$ & 0.89 \\
Anxiety & 0.04 & 5.38 & 1 & 0.020 & 379.65 & $0.146-0.194$ & 1.04 \\
Depression & 0.05 & 8.89 & 1 & 0.003 & 374.10 & $0.161-0.215$ & 1.05 \\
\hline
\end{tabular}

The results showed that the overall model correctly predicted heart problems among participants, $\chi^{2}(d f=4)=54.24, p<0.001$. This model has a good fit, $-2 \log$ likelihood $=374.10$, Hosmer and Lemeshow, $\chi^{2}(d f=8)=8.79, p>0.05$.

The results of the Cox-Snell and the Nagelkerke $R^{2}$ indicated that age accounted for $5.6-7.5 \%$ of the variance in heart problems. Social inhibition accounted for 
another $5.0-6.6 \%$ of the variance. Anxiety added $4.0-5.3 \%$, and depression added $1.5-2.1 \%$ to the variance in having heart problems. Overall, the model accounted for $16.1-21.5 \%$ of the variance in having heart problems. Finally, the model correctly classified $69.3 \%$ of the 'having no heart problems' cases and $66 \%$ of the 'having heart problems' cases. Overall, this model had a success rate of $68 \%$.

A hierarchical regression analysis was conducted to determine the power of sociodemographic variables (Step 1: age and gender), type of heart condition (Step 2: blood vessel diseases, coronary artery disease, arrhythmias, angina, or stroke, and Type D personality), (Step 3: negative affectivity vs social inhibition) in predicting the degree of distress or anxiety experienced by subjects (see Table 2). Two factors emerged as significant predictors of anxiety: type of heart condition, $\beta=0.22, p<$ 0.001 , and Type $\mathrm{D}$ personality, $\beta=0.49, p<0.001$. In this analysis, type of heart condition accounted for about $4 \%$ of the variance; Type $\mathrm{D}$ personality accounted for $24 \%$ of the variance. In this model, the combination of both significant factors accounted for $28 \%$ of the variance.

Table 2

Type of heart condition and Type D personality as predictors of depression and anxiety: hierarchical regression analysis

\begin{tabular}{lccccc}
\hline Factor & $R$ & $R^{2}$ & $\beta$ & $F$ & $p$ \\
\hline Anxiety & 0.20 & 0.04 & 0.22 & 12.52 & $<0.001$ \\
Type of Heart Condition & 0.53 & 0.28 & 0.49 & 59.41 & $<0.001$ \\
Type D Personality & & & & & \\
Depression & 0.24 & 0.06 & 0.26 & 18.00 & $<0.001$ \\
Type of Heart Condition & 0.48 & 0.24 & 0.42 & 46.42 & $<0.001$ \\
Type D Personality & & & & & \\
\hline
\end{tabular}

Similar trends were detected with distress of depression, $F(2.306)=46.42, p<$ 0.001 ; type of heart condition, $\beta=0.26, p<0.001$; and Type $\mathrm{D}$ personality, $\beta=0.42$, $p<0.001$. Type of heart condition accounted for about $6 \%$ of the variance, whereas Type D personality accounted for $18 \%$ of the variance, and the joint contribution of both factors accounted for $24 \%$.

However, when an analysis was conducted using dimensions of Type D personality (social inhibition and negative affectivity), only negative affectivity was detected as a significant predictor of anxiety, accounting for $32 \%$ of the variance; social inhibition emerged as a nonsignificant factor, $\beta=0.05, p>0.05$. For depression, 
type of heart condition again accounted for $6 \%$ of the variance. However, of the total effect of Type D personality (18\%), $1 \%$ percent was attributed to the social inhibition factor, and the vast majority of the variance (17.85\%) was assigned to negative affectivity.

\section{Discussion}

Cardiovascular health has drawn increasing attention in Jordan. Our research has uncovered that social inhibition, anxiety, and depression are risk factors for the development of cardiovascular disorders, confirming the previous research of European and American samples. Further, the results negate the dichotomy between psychological and personality dimensions of well-being.

Specifically, age accounted for $5.6-7.5 \%$ of the variance in heart problems, social inhibition accounted for another 5.0-6.6\%, and anxiety accounted for 4.0-5.3\%. Overall, the model accounted for $16.1-21.5 \%$ of the variance in cardiac disorders. The model correctly classified $69.3 \%$ of the healthy sample and $66 \%$ percent of the target group sample. Accordingly, this model had a success rate of $68 \%$. Older participants with higher levels of social inhibition, anxiety, and depression were more likely to experience cardiovascular disease. The results highlighted the role of social inhibition in the progression and prognosis of cardiovascular disease.

The results, however, did not indicate the direction and the causal inferences. More advanced statistical analyses are needed to determine the antecedent. Bidirectionality among Type $\mathrm{D}$ personality, depression, anxiety, and cardiac conditions also may occur. Hierarchical regression analysis may provide a plausible explanation to the bidirectionality among the variables. The analysis of hierarchical regression demonstrated that the type of vascular condition and Type D personality (particularly negative affectivity) were significant predictors of distress from depression. In particular, the type of vascular condition accounted for approximately $4 \%$ of the variance in anxiety indices and $24 \%$ for Type D personality. Similar patterns were detected with depression. Thus, one strategy of health-promotive behavior programs should be to target the main effect associated with these risk factors. Programs targeting cardiovascular patients should introduce additional assets that function as protective factors, to compete or counterbalance the negative effects of risk factors, thereby allowing the patient to maintain normative heart condition functioning. Health promotive programs may be designed as either proactive or reactive programs.

Furthermore, it is difficult to determine whether the level of depression and anxiety associated with cardiovascular disease is independent of Type D personality. Depression and anxiety may be partially assigned to the negative affectivity dimension of Type D personality. The link between depression and anxiety has been well 
established in heart disease patients (KupPer \& Denollet 2016; Molina-Leyva et al. 2015). Through mechanisms of physiological hyperreactivity, immune activation, and poor health behaviors, Type D personality can adversely shape health conditions and catalyze the development of heart disease (Gilmour \& Williams 2012). This personality trait can impede satisfaction with quality of life (SogARo et al. 2015), directly affecting self-esteem (Huis et al. 2011).

Patients with Type D personality are likely to experience higher levels of perceived stress as they use more passive and maladaptive avoidance coping styles (Polman et al. 2010). Consequently, this population fails to utilize social support - an essential method known as evidence-based techniques in minimizing psychological stress (GINTING et al. 2016). These findings call for interventions targeting internal and external resources, such as improving patients' lifestyle choices. Other internal health-promotive resources to consider for treatment involve improving patients' self-esteem, self-efficacy, and self-regulations. These efforts are potent interventions to help deter or neutralize the adverse effects of risk factors linked to depression and anxiety.

Finally, given the collective nature of Jordanian society and the stigma associated with seeking mental health therapies, intervention efforts must include community-based empowerment strategies. Greater awareness of cardiovascular disease risk factors is needed for individuals to recognize their personal risk, adapt health-promotive preventative behaviors, and seek necessary therapies.

\section{Summary}

According to the World Health Organization (2017), 31\% of global deaths are attributed to cardiovascular disease, cementing it as the main cause of death in the human population. In the past, the study of cardiac and vascular diseases remained embedded in the medical paradigm. However, studies suggesting the vital function of behavior in the etiology of such diseases have increased exponentially, predicting the joint determination of behavioral and biological processes in the pathogenesis of cardiovascular diseases (RosENGREN et al. 2004). Behavioral cardiology has expanded as a field within cardiovascular medicine, with a broad repertoire of literature available on the epidemiological and pathophysiological implications of psychosocial risk factors in the etiology of cardiac diseases.

The current study is nested in the risk framework, giving prominence to personality risk factors predisposing or placing individuals at higher risk status for CHD. The study's findings confirm the essential nature of the psychological and personality risk/protective factor framework. The results also identify processes that mediate between risk factors and cardiovascular disease. 
To counteract the risk factors associated with depression, anxiety, and the traits of Type D personality, practitioners could cultivate the patient's health-promotive self-efficacy, self-control, self-regulation, and other critical coping strategies to neutralize the damaging effects of these risk factors. Of course, some individuals may have the potential to alter disease etiology by balancing risk and protective processes.

This study has inherent limitations as a cross-sectional study, and longitudinal research designs are preferred in capturing the continuity and discontinuity of the adverse effects of risk factors in the progression and prognosis of cardiovascular disease. More sophisticated analysis is needed to understand not only personality and social risk factors, but also the processes involved in the course of cardiovascular disease. The supplementary role of protective factors, as well as the reciprocation between risk and protective factors, are two domains that should guide health-promotive intervention programs to moderate the adverse trajectories of risk factors. In addition, essential intervention should incorporate both individual therapeutic modalities and culturally responsive community outreach programs to empower patients at risk of heart disease.

\section{References}

Alansari, B.M. (2005) 'Beck Depression Inventory (BDI-II) Items Characteristics among Undergraduate Students of Nineteen Islamic Countries', Social Behavior and Personality: An international journal 33(7), 675-84 (https://doi.org/10.2224/ sbp.2005.33.7.675).

Al-DA'AsIn, K. (2004) Psychometric Characteristics of Beck II List of Depression (BDI-II) in the Jordanian Environment (MA thesis, Mutah University, Karak, Jordan).

Al-Musawi, N.M. (2001) 'Psychometric Properties of the Beck Depression Inventory-II with University Students in Bahrain', Journal of Personality Assessment 77(3), 568-79 (https://doi.org/10.1207/S15327752JPA7703_13).

Al-OMari, A. \& M. Al-Hussaini (2017) 'Research Ethics Governance in the Arab Region: Jordan' in H. Silverman eds., Research Ethics in the Arab Region. Research Ethics Forum, Volume 5 (Cham: Springer) (https://doi.org/10.1007/9783-319-65266-5_21).

Angermann, C.E., G. Gelbrich, S. Störk, M. Schowalter, J. Deckert, G. ERTL \& H. Faller (2011) 'Somatic Correlates of Comorbid Major Depression in Patients with Systolic Heart Failure', International Journal of Cardiology 147(1), 66-73 (https://doi.org/10.1016/j.ijcard.2009.07.044).

BeCK, A.T., R.A. STEER \& G.K. Brown (1996) Beck Depression Inventory-II (BDI-II) (San Antonio, TX: Psychological Corporation). 
Bоoтh, L. \& L. Williams (2015) 'Type D Personality and Dietary Intake: The Mediating Effects of Coping Style', Journal of Health Psychology 20(6), 921-27 (https://doi.org/10.1177/1359105315573433).

Bunevicius, A., J. Brozaitiene, M. Staniute, V. Gelziniene, I. Duoneliene, V.J.M. Pop, R. Bunevicius \& J. Denollet (2014) 'Decreased Physical Effort, Fatigue, and Mental Distress in Patients with Coronary Artery Disease: Importance of Personality-Related Differences', International Journal of Behavioral Medicine 21, 240-47 (https://doi.org/10.1007/s12529-013-9299-9).

De Hert, M., J. Detraux \& D. Vancampfort (2018) 'The Intriguing Relationship Between Coronary Heart Disease and Mental Disorders', Dialogues in Clinical Neuroscience 20(1), 31-40.

Denollet, J. (2005) 'DS14: Standard Assessment of Negative Affectivity, Social Inhibition, and Type D Personality', Psychosomatic Medicine 67(1), 89-97 (https://doi.org/10.1097/01.psy.0000149256.81953.49).

Denollet, J., S.U. Sys \& D.L. Brutsaert (1995) 'Personality and Mortality After Myocardial-Infarction', Psychosomatic Medicine 57(6), 582-91 (https://doi. org/10.1097/00006842-199511000-00011).

Denollet, J., C.J. VRints \& V.M. ConRaAds (2008) 'Comparing Type D personality and Older Age as Correlates of Tumor Necrosis Factor-a Dysregulation in Chronic Heart Failure', Brain, Behavior, and Immunity 22(5), 736-43 (http:// doi.org/10.1016/j.bbi.2007.10.015).

Emons, W.H.M., R.R. MeiJeR \& J. Denollet (2007) 'Negative Affectivity and Social Inhibition in Cardiovascular Disease: Evaluating Type-D Personality and its Assessment Using Item Response Theory', Journal of Psychosomatic Research 63(1), 27-39 (https://doi.org/10.1016/j.jpsychores.2007.03.010).

Gilmour, J. \& L. Williams (2012) 'Type D Personality Is Associated with Maladaptive Health-Related Behaviours', Journal of Health Psychology 17(4), 471-78 (https://doi.org/10.1177/1359105311423117).

Ginting, H., M. van De Ven, E.S. Becker \& G. NÄring (2016) 'Type D Personality Is Associated with Health Behaviors and Perceived Social Support in Individuals with Coronary Heart Disease', Journal of Health Psychology 21(5), 727-37 (https://doi.org/10.1177/1359105314536750).

Huis in't Veld, E.M.J., A.J.J.M Vingerhoets \& J. Denollet (2012) 'Attachment Style and Self-Esteem: The Mediating Role of Type D Personality', Personality and Individual Differences 50(7), 1099-1103 (https://doi.org/10.1016/j. paid.2011.01.034).

International Committee of Medical Journal Editors (2018) Recommendations for the Conduct, Reporting, Editing, and Publication of Scholarly Work in Medical Journals, retrieved 25 Aug 2021 from http://www.icmje.org/recommendations/. 
KupPer, N. \& J. Denollet (2018) 'Type D Personality as a Risk Factor in Coronary Heart Disease: A Review of Current Evidence', Current Cardiology Reports 20, 104 (https://doi.org/10.1007/s11886-018-1048-x).

Lilienfeld, A.M. \& D.E. LiLienfeld (1980) Foundations of Epidemiology (New York: Oxford UP).

Lim, H.E., M.-S. Lee, Y.-H. Ko, Y.-M. Park, S.-H. Joe, Y.-K. Kim, C. Han, H.-Y. Lee, S.S. Pedersen \& J. Denollet (2011) 'Assessment of the Type D Personality Construct in the Korean Population: A Validation Study of the Korean DS14', Journal of Korean Medical Science 26(1), 116-23 (https://doi.org/10.3346/ jkms.2011.26.1.116).

Molina-Leyva, A., I. Caparros-delMoral, J.C. Ruiz-Carrascosa, R. NaranjoSintes \& J.J. Jimenez-Moleon (2015) 'Elevated Prevalence of Type D (Distressed) Personality in Moderate to Severe Psoriasis Is Associated with Mood Status and Quality of Life Impairment: A Comparative Pilot Study', Journal of the European Academy of Dermatology and Venereology 29(9), 1710-17 (https://doi.org/10.1111/jdv.12960).

Moraska, A.R., A.M. Chamberlain, N.D. Shah, K.S. Vickers, T.A. Rummans, S.M. Dunlay, J.A. Spertus, S.A.Weston, S.M. McNallan, M.M. Redfield \& V.L. Roger (2013) 'Depression, Healthcare Utilization, and Death in Heart Failure', Circulation: Heart Failure 6(3), 387-94 (http://doi.org/10.1161/circheartfailure.112.000118).

Nefs, G., J. Speight, F. Pouwer, V. Pop, M. Bot \& J. Denollet (2015) 'Type D Personality, Suboptimal Health Behaviors and Emotional Distress in Adults with Diabetes: Results from Diabetes MILES-The Netherlands', Diabetes Research and Clinical Practice 108(1), 94-105 (https://doi.org/10.1016/j.diabres.2015.01.015).

Oliva, F., E. Versino, L. Gammino, N. Colombi, L. Ostacoli, S. Carletto, P.M. FurLAN \& R.L. PICCI (2016) 'Type D Personality and Essential Hypertension in Primary Care: A Cross-Sectional Observational Study Within a Cohort of Patients Visiting General Practitioners', Journal of Nervous and Mental Disease 204(1), 43-48 (https://doi.org/10.1097/NMD.0000000000000421).

Polman, R., E. Borkoles \& A.R. Nicholls (2010) 'Type D Personality, Stress, and Symptoms of Burnout: The Influence of Avoidance Coping and Social Support', British Journal of Health Psychology 15(3), 681-96 (https://doi. org/10.1348/135910709X479069).

Razzini, C., F. Bianchi, R. Leo, E. Fortuna, A. Siracusano \& F. Romeo (2008) 'Correlations Between Personality Factors and Coronary Artery Disease: From Type A Behaviour Pattern to Type D Personality', Journal of Cardiovascular Medicine 9(8), 761-68 (https://doi.org/10.2459/JCM.0b013e3282f39494).

Rosengren, A., S. Hawken, S. Ôunpuu, K. Sliwa, M. Zubaid, W.A. Almahmeed, K.N. Blackett, C. Sitthi-Amorn, H. Sato \& S. Yusuf (2004) 'Association of Psychosocial Risk Factors with Risk of Acute Myocardial Infarction in 11,119 
Cases and 13,648 Controls From 52 Countries (The INTERHEART Study): Case-Control Study', The Lancet 364(9438), 953-62 (https:/doi.org/10.1016/ s0140-6736(04)17019-0).

Sogaro, E., F. Schininà, C. Burgisser, F. Orso, R. Pallante, T. Aloi, D. Vanni, A. Pazzagli \& F. Fattirolli (2010) 'Type D Personality Impairs Quality of Life, Coping and Short-Term Psychological Outcome in Patients Attending an Outpatient Intensive Program of Cardiac Rehabilitation', Monaldi Archives for Chest Disease 74(4), 181-91 (https://doi.org/10.4081/monaldi.2010.259).

Spielberger, C.D. \& S.J. Sydeman (1994) 'State-Trait Anxiety Inventory and State-Trait Anger Expression Inventory’ in M.E. MARuish ed., The Use of Psychological Testing for Treatment Planning and Outcome Assessment (Hillsdale, NJ: Erlbaum) 292-321.

Spielberger, C.D., R.L. Gorsuch \& R.E. Lushene (1970) Manual for the State-Trait Anxiety Inventory (Palo Alto, CA: Consulting Psychologists Press).

Spielberger, C.D., R.L. Gorsuch \& R.E. Lushene (2005) Manual for the State-Trait Anxiety Inventory, trans. A.R. Al-BuhaIRI (Cairo: The Egyptian Renaissance Library).

Svansdottir, E., H.D. Karlsson, T. Gudnason, D.T. Olason, H. Thorgilsson, U. Sigtryggsdottir, E.J. Sijbrands, S.S. Pedersen \& J. Denollet (2012) 'Validity of Type D Personality in Iceland: Association with Disease Severity and Risk Markers in Cardiac Patients', Journal of Behavioral Medicine 35, 155-66 (https://doi.org/10.1007/s10865-011-9337-5).

Wiencierz, S. \& L. Williams (2017) 'Type D Personality and Physical Inactivity: The Mediating Effects of Low Self-Efficacy', Journal of Health Psychology 22(8), 1025-34 (https://doi.org/10.1177/1359105315622557).

Williams, L., R.C. O’Connor, N. GrubB \& R. O’Carroll (2011) 'Type D Personality Predicts Poor Medication Adherence in Myocardial Infarction Patients', Psychology \& Health 26(6), 703-12 (https://doi.org/10.1080/08870446.2010. 488265).

World Health Organization (2021) Cardiovascular diseases (CVDs), retrieved 25 Aug 2021 from http://www.who.int/news-room/fact-sheets/detail/cardiovascular-diseases-(cvds).

World Medical Association (2013) WMA declaration of Helsinki-Ethical principles for medical research involving human subjects (Amended by the 64th WMA General Assembly, Fortaleza, Brazil, October 2013), retrieved 25 Aug 2021 from https://www.wma.net/policies-post/wma-declaration-of-helsinki-ethical-principles-for-medical-research-involving-human-subjects/.

Wu, J.R., E.K. Song \& D.K. Moser (2015) 'Type D Personality, Self-Efficacy, and Medication Adherence in Patients with Heart Failure-A Mediation Analysis', Heart \& Lung 44(4), 276-81 (https://doi.org/10.1016/j.hrtlng.2015.03.006). 